\title{
Modified Ring Power Divider Using Stepped-Impedance Resonator
}

\author{
Rabih Barakat $^{1}$ (D), Vahe Nerguizian ${ }^{1}$ (D), Djilali Hammou ${ }^{2}$ (D), Serioja Ovidiu Tatu ${ }^{2}$ (1) \\ ${ }^{I}$ Ecole de Technologie Superieure 1100, Notre-Dame St W., Montreal, Quebec, H3C1K3, Canada \\ ${ }^{2}$ INRS-EMT, University of Quebec 800, de La Gauchetiere W., Suite 6900, Montreal, Quebec, \\ H5AlK6, Canada \\ rabih.barakat@polymtl.ca,vahe.nerguizian@etsmtl.ca,hammou@emt.inrs.ca, tatu@em t.inrs.ca
}

\begin{abstract}
In this paper, the design of a modified ring power divider dedicated for wideband communication or measurement systems is presented. The proposed power divider is built by using a conventional ring power divider and an additional SteppedImpedance Resonator (SIR). The even-odd mode analysis is used to derive the design equations. In order to observe the bandwidth improvement, a comparative study between the designed device and the previously reported ring power divider is conducted. The electromagnetic (EM) simulated results show that the proposed divider has a fractional bandwidth of $90.2 \%$ at the center frequency of $3.17 \mathrm{GHz}$. The measurement results of the fabricated prototype demonstrate high performance over the considered operational bandwidth from $1.7 \mathrm{GHz}$ to $4.64 \mathrm{GHz}$ with a return loss lower than -10 dB while maintaining a good insertion loss and a good isolation between the output ports. The maximum amplitude imbalance is better than $0.21 \mathrm{~dB}$ and the phase imbalance is better than 2.5 degrees between the output signals. The simulation and measurement results are in good agreement over the whole band of interest.
\end{abstract}

Index Terms - Microstrip, Ring Power divider, Stepped-impedance resonator (SIR); Wideband.

\section{INTRODUCTION}

Power dividers are extremely useful microwave components whose principal function is to equally split the incident signal between the two outputs while maintaining good isolation between them. They are used in different applications including measurement systems as well as antenna distribution circuits, communication and radar systems. Many efforts have been recently made to design wideband power dividers by opportunely modifying the classical Wilkinson structure [1].

The most usual way to make this device wideband is by adding two or more sections to the conventional structure [2]-[4] or by adding open-circuited stubs [5]-[6]. However, these structures add more circuit area to the conventional design. Other works, reported in the literature, propose broadband Wilkinson power dividers such as in [7] where double-sided parallel-strip line technique using similar structure at both the top and bottom layers with three isolation resistors at both layers is proposed. In [8], the proposed power divider using microstrip-to-slotline coupled structure and a top and bottom layers shows good performance over a $68.6 \%$ bandwidth from 2.3 to $4.7 \mathrm{GHz}$. Another 
broadband power divider achieving good in-band responses across a $62 \%$ fractional bandwidth was proposed in [9] by arranging two open-circuited stubs and three coupled-line sections around the ring resonator. However, these power dividers require complex design configuration due to the use of different layers or coupled-line sections which makes the fabrication process more complicated.

Recently, a compact ring power divider has been proposed in [10]. It introduces a circular layout with the addition of two half-wave transmission lines to connect the $100 \Omega$ isolation resistor, converting the layout in a ring circuit of $1.5 \lambda_{\mathrm{g}}$ length. The corresponding electric lengths $\theta_{1}$ and $\theta_{2}$ are $\pi / 2$ and $\pi$, respectively, and the normalized ring characteristic impedance $\mathrm{z}_{01}$ (which is equal to $\mathrm{z}_{02}$ ) is found to be $\sqrt{2}$ [10]. The circuit has been rebuilt to operate at the center frequency of $3.12 \mathrm{GHz}$ as shown in Fig. 1.

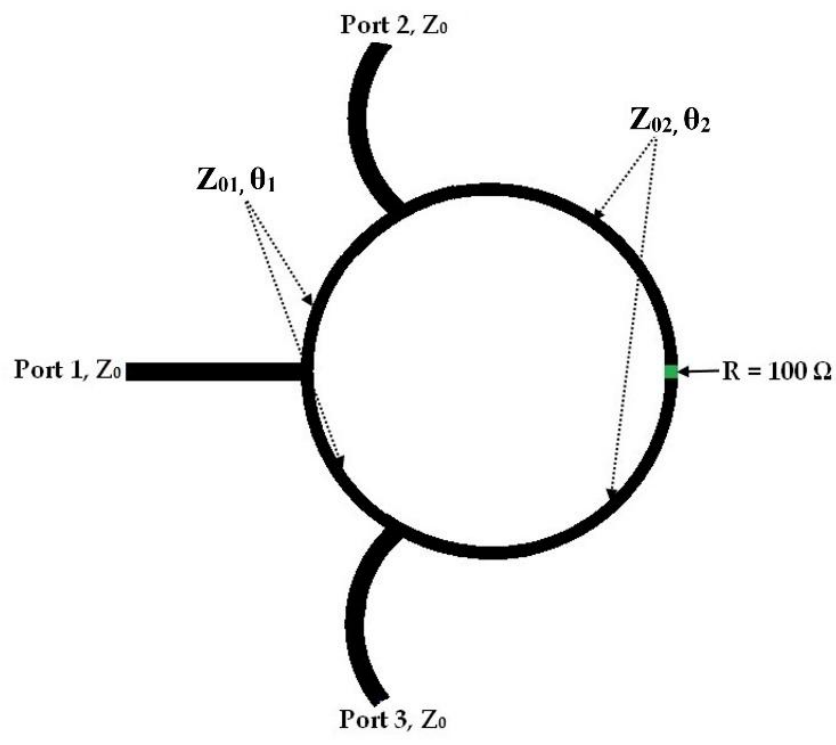

Fig. 1. Layout of the ring power divider proposed in [10].

This circuit is offering very good performance over a wide bandwidth compared to the traditional Wilkinson power divider. In addition, this design had shown more stability and larger bandwidth than previous implementations among valid solutions. Furthermore, this circuit has a size reduction of around $20 \%$ compared to classical Wilkinson power divider and improved performances in terms of RF input/output matching and isolation between the output RF ports [11].

In this work, a modified ring power divider operating in the frequency band from $1.7 \mathrm{GHz}$ to 4.64 $\mathrm{GHz}$ is introduced and it consists of the same circular structure as in [10] with the addition of a stepped-impedance resonator (SIR) to the old structure. The circuit in Fig. 2 represents the layout of the proposed ring power divider. For this structure, the simulation results show that the fractional bandwidth has a significant increase of $27.2 \%$ compared to the old structure as it will be seen in section II and this without affecting the circuit size since the added element is inside the ring structure. 


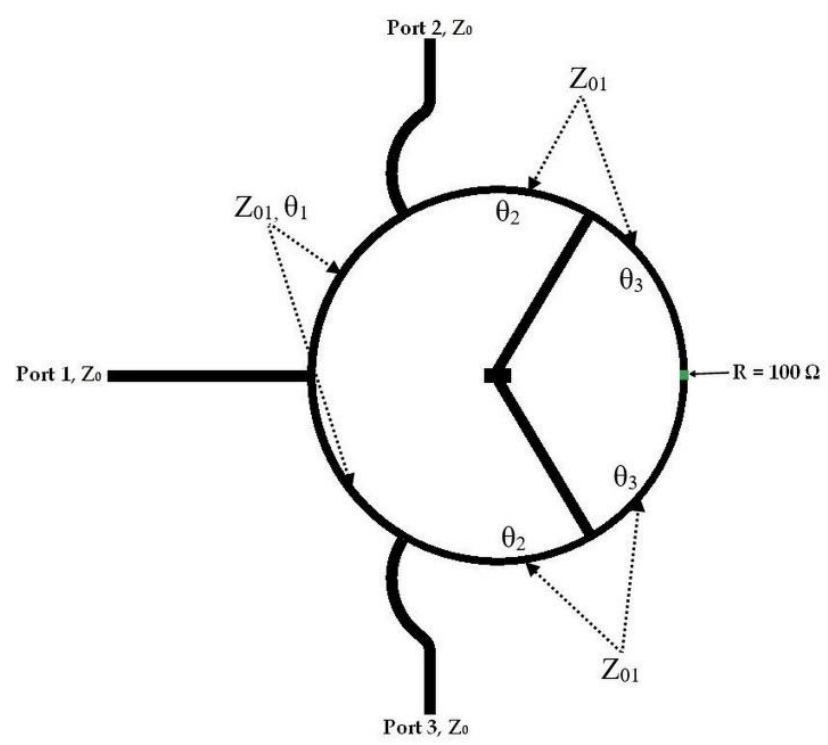

Fig. 2. Layout of the proposed ring power divider.

\section{DESIGN AND SIMULATIONS}

The circuit diagram of the proposed wideband ring power divider is shown in Fig. 3. It consists of six transmission lines of characteristic impedances $\left(\mathrm{Z}_{01}, \mathrm{Z}_{02}\right.$, and $\left.\mathrm{Z}_{03}\right)$, a Stepped-Impedance Resonator (SIR), and an isolation resistor $\mathrm{R}=100 \Omega$.

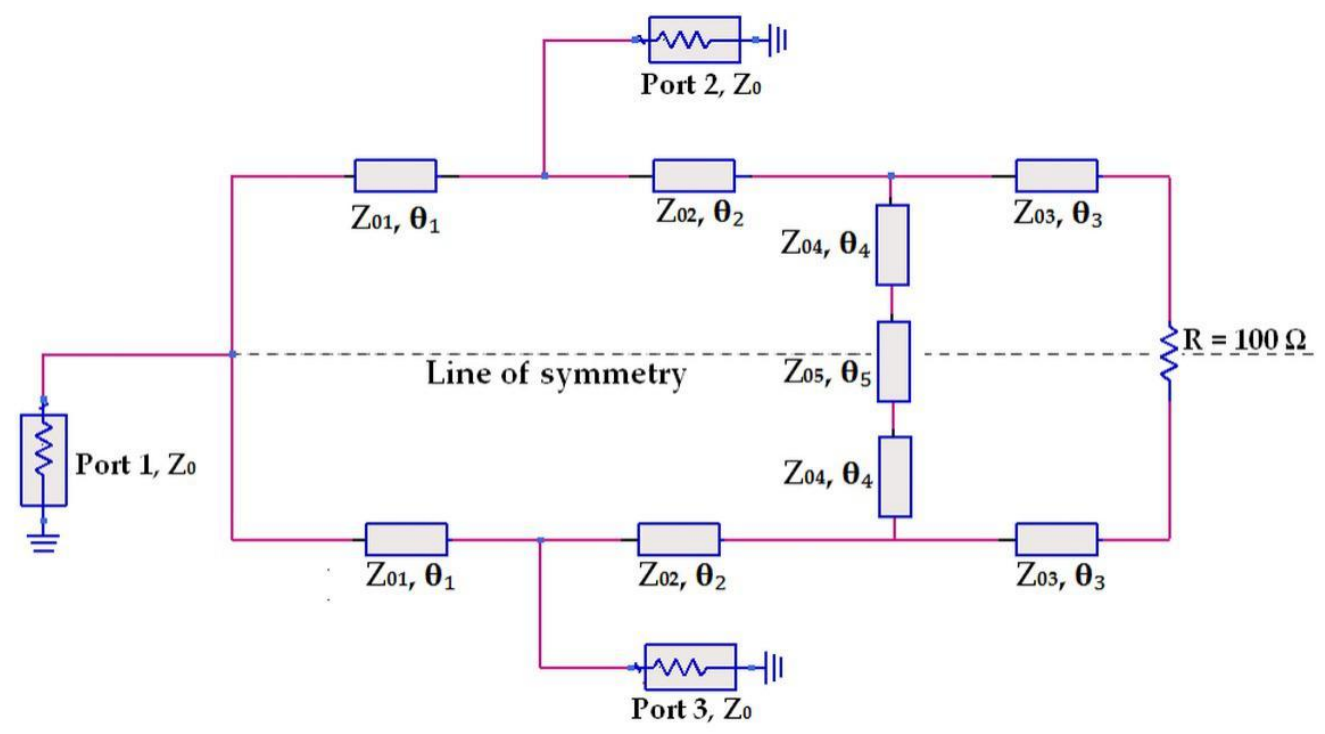

Fig. 3. Proposed structure of the modified ring power divider.

Microstrip resonators have been widely used in the design of microwave filters with wide stopband characteristics [12]-[14]. In addition, Stepped-impedance resonators (SIRs) have been found advantageous in the design of microstrip bandpass filters with good stopband performance [15]-[17]. A typical SIR is used to modify the structure of the ring power divider and is built by joining three microstrip-line sections of different characteristic impedances $Z_{04}$ and $Z_{05}$ as shown in Fig. 4. The microstrip line at the center has a characteristic impedance of $Z_{05}$ with an electrical length of $\theta_{5}$ whereas the identical lines at the two sides both have a characteristic impedance of $\mathrm{Z}_{04}$ with an Brazilian Microwave and Optoelectronics Society-SBMO received 23 June 2019; for review 27 June 2019; accepted 18 Nov 2019 
electrical length of $\theta_{4}$.

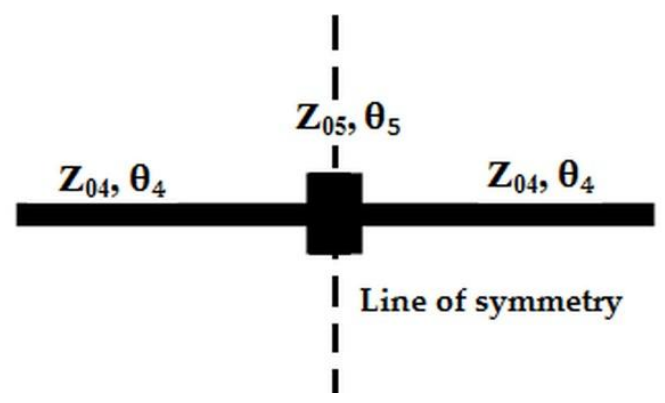

Fig. 4. Stepped impedance resonator (SIR) used structure.

As described in [16], the conditions for determining the resonance frequencies of a typical stepped impedance resonator (SIR) are given as:

$$
\begin{array}{ll}
K=\tan \left(\theta_{5} / 2\right) \cdot \tan \theta_{4} & \text { (for odd mode) } \\
K \tan \left(\theta_{5} / 2\right)=-\tan \theta_{4} & \text { (for even mode) }
\end{array}
$$

Where $\mathrm{K}=Z_{04} / Z_{05}$ is the impedance ratio of the SIR.

Let's define the stepped percentage $\alpha$ of the SIR as $\alpha=2 \theta_{4} / \theta_{t}$ where $\theta_{t}=\left(2 \theta_{4}+\theta_{5}\right)$ is the total length of the SIR. Then, equations (1) and (2) can be expressed as:

$$
\begin{aligned}
K \cdot \cot \left(\frac{\alpha \cdot \theta_{t}}{2}\right) & =\tan \left(\frac{(1-\alpha) \cdot \theta_{t}}{2}\right) & & \text { (for odd mode) } \\
K \cdot \cot \left(\frac{\alpha \cdot \theta_{t}}{2}\right) & =-\cot \left(\frac{(1-\alpha) \cdot \theta_{t}}{2}\right) & & \text { (for even mode) }
\end{aligned}
$$

Fig. 5 shows the resonant electric length of the first spurious response mode with different impedance ratios $\mathrm{K}=1,1.5,2,3$ as a parameter. By properly choosing different combinations of $\alpha$ and $\mathrm{K}$ values, the first two resonant modes can be combined together to create a wide passband [16].

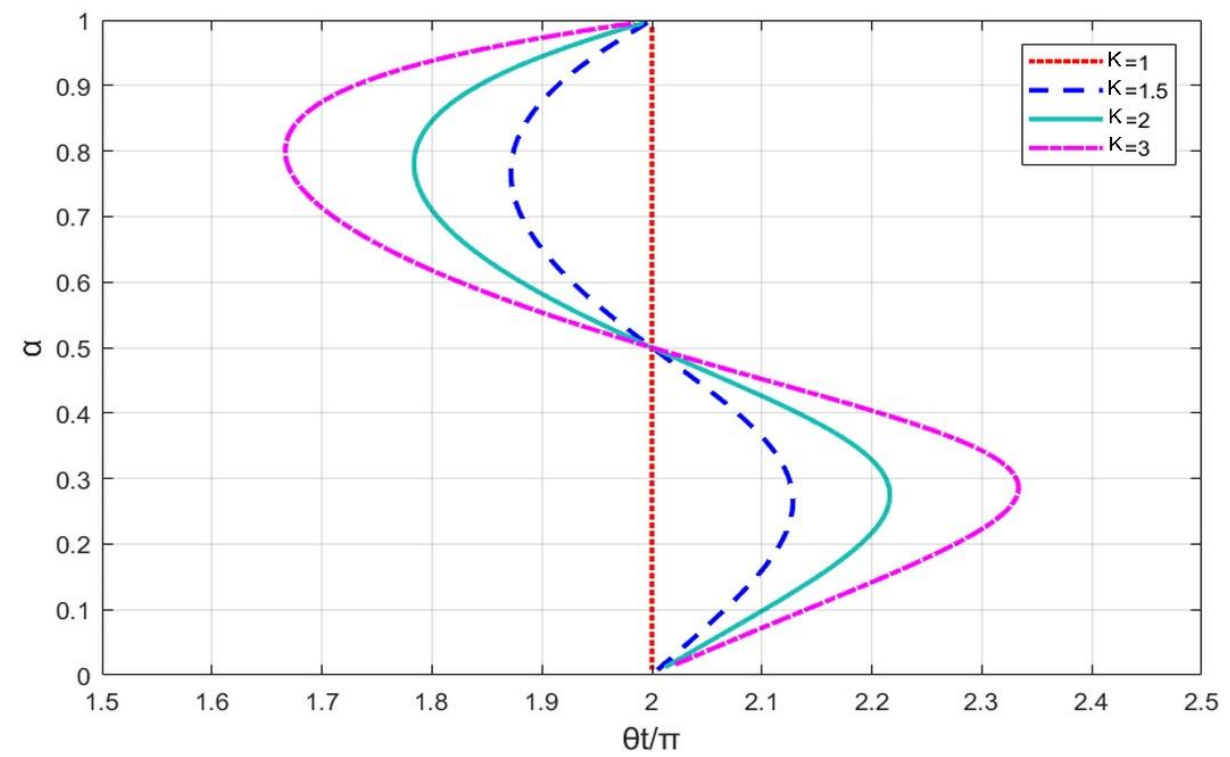

Fig. 5. Resonant electric length $\theta_{\mathrm{t}}$ versus stepped percentage $\alpha$ with different impedance ratios K. 
The structure of the proposed ring power divider is symmetric; thus, we can apply the even-odd mode analysis [18] in order to analyze the divider parameters. In this study, the known parameters as defined in the previous analysis [10] are the normalized characteristic impedances $\mathrm{z}_{01}=\mathrm{z}_{02}=\mathrm{z}_{03}=\mathrm{z}_{04}=$ $\sqrt{2}$; and $\theta_{1}=\pi / 2$ and $\left(\theta_{2}+\theta_{3}\right)=\pi$. The following analysis is performed in order to find the main characteristics of the added element to the old ring structure.

\section{A. Even -mode analysis}

In the even mode, ports 2 and 3 are excited by signals of the same magnitude and phase, which introduces a virtual open-circuit along the line of symmetry. By introducing the same voltages at the plane of symmetry there is no current flow through the line of symmetry and the presence of open circuits allows for eliminating the resistance $\mathrm{R}$ in the equivalent circuit as shown in Fig. 6 . The calculation of the input impedance for the even-mode $Z_{\text {ine }}$ viewed from port 2 corresponds to the equivalent impedances $Z_{\text {eq1e }}$ and $Z_{\text {eq2e }}$ connected in parallel.

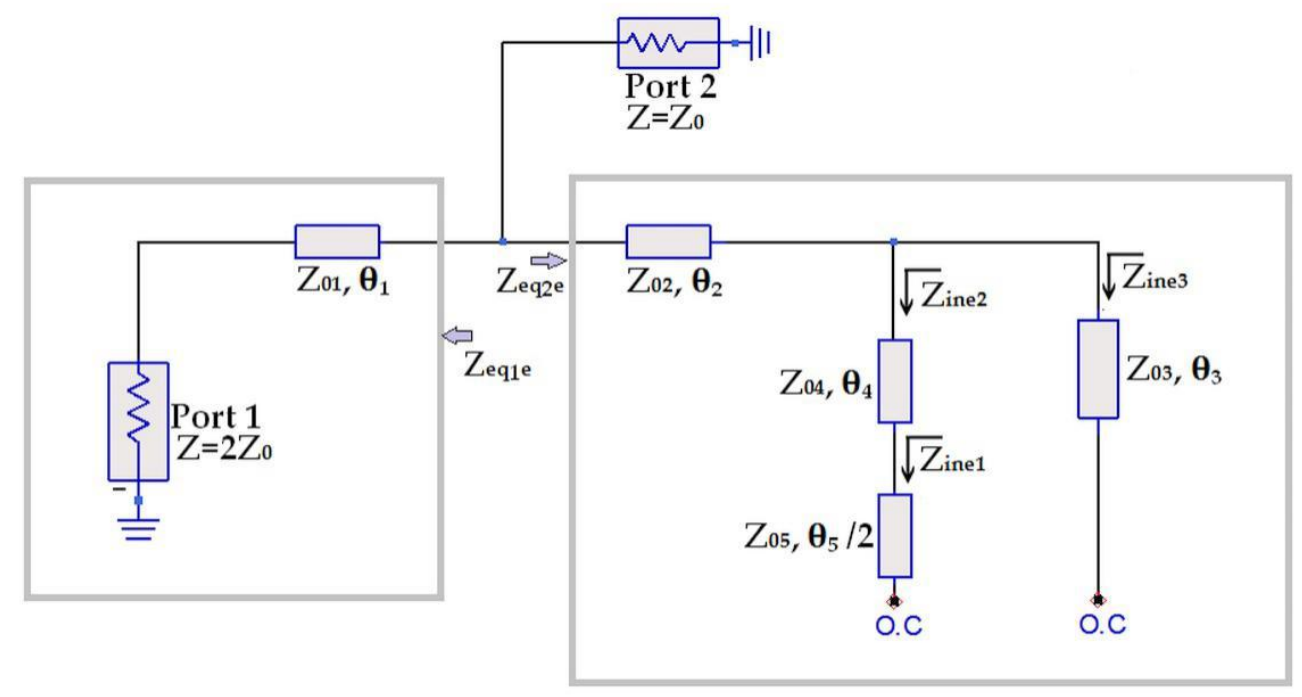

Fig. 6. Equivalent circuit of the proposed ring divider for odd-mode.

From the transmission line theory, we can easily calculate $\mathrm{Z}_{\mathrm{eq} 1 \mathrm{e}}$ as the input impedance of a quarterwave transmission line terminated by $2 Z_{0}$ :

$$
Z_{\text {eq1e }}=\frac{Z_{01}^{2}}{2 Z_{0}}=\frac{2 Z_{0}^{2}}{2 Z_{0}}=Z_{0}
$$

Referring to Fig. 6, the input impedances $Z_{\text {ine } 1}$ and $Z_{\text {ine2 }}$ can be expressed as follows

$$
\begin{gathered}
Z_{\text {ine } 1}=-j Z_{05} \cot \left(\frac{\theta_{5}}{2}\right) \\
Z_{\text {ine } 2}=\frac{Z_{04}\left(Z_{\text {ine } 1}+j Z_{04} \tan \theta_{4}\right)}{\left(Z_{04}+j Z_{\text {ine } 1} \tan \theta_{4}\right)} \\
Z_{\text {ine } 2}=\frac{-j Z_{04}\left(Z_{05} \cot \left(\theta_{5} / 2\right)-Z_{04} \tan \theta_{4}\right)}{Z_{04}+Z_{05} \cot \left(\theta_{5} / 2\right) \tan \theta_{4}}
\end{gathered}
$$

Brazilian Microwave and Optoelectronics Society-SBMO received 23 June 2019; for review 27 June 2019; accepted 18 Nov 2019 
In a similar way, the admittance $\mathrm{Y}_{\text {ine3 }}$ can be expressed as the input admittance of an open-circuited transmission line.

$$
Y_{\text {ine3 }}=\frac{1}{Z_{\text {ine } 3}}=j Y_{03} \tan \theta_{3}
$$

Let $Z_{X}$ be the parallel combination of these impedances $Z_{\text {ine2 }}$ and $Z_{\text {ine3 }}$, then the equivalent admittance $Y_{X}$ can be written as:

$$
Y_{X}=Y_{\text {ine3 }}+Y_{\text {ine } 2}=j\left[Y_{03} \tan \theta_{3}+Y_{04} \frac{\left(Z_{04}+Z_{05} \cot \left(\theta_{5} / 2\right) \tan \theta_{4}\right)}{\left(Z_{05} \cot \left(\theta_{5} / 2\right)-Z_{04} \tan \theta_{4}\right)}\right]
$$

The equivalent impedance $Z_{\text {eq2e }}$ can be expressed as the input impedance of a transmission line terminated by $Z_{\mathrm{X}}$ :

$$
Z_{e q 2 e}=\frac{Z_{02}\left(Z_{X}+\mathrm{j} Z_{02} \tan \theta_{2}\right)}{\left(Z_{02}+j Z_{X} \tan \theta_{2}\right)}
$$

Since the output is matched, the input admittance for the even-mode $Y_{\text {ine }}$ viewed from port 2 should be equal to $\mathrm{Y}_{0}=1 / \mathrm{Z}_{0}$ :

$$
Y_{\text {ine }}=\frac{1}{Z_{\text {eq1e }}}+\frac{1}{Z_{\text {eq2e }}}=\frac{1}{Z_{0}}
$$

By replacing $Z_{\text {eq1e }}$ and $Z_{\text {eq2e }}$ in (12) with their values in (5) and (11), this will result in

$$
\frac{\left(Z_{02}+j Z_{X} \tan \theta_{2}\right)}{Z_{02}\left(Z_{X}+\mathrm{j} Z_{02} \tan \theta_{2}\right)}=0
$$

This yields to

$$
Y_{X}=-j Y_{02} \tan \theta_{2}
$$

Comparing (10) and (14), and since $Y_{03}=Y_{02}$; we have

$$
Y_{03}\left(\tan \theta_{3}+\tan \theta_{2}\right)+Y_{04} \frac{\left(Z_{04}+Z_{05} \cot \left(\theta_{5} / 2\right) \tan \theta_{4}\right)}{\left(Z_{05} \cot \left(\theta_{5} / 2\right)-Z_{04} \tan \theta_{4}\right)}=0
$$

By using the trigonometric identity

$$
\left(\tan \theta_{3}+\tan \theta_{2}\right)=\left(\tan \left(\theta_{3}+\theta_{2}\right)\right)\left[1-\tan \theta_{3} \tan \theta_{2}\right]
$$

Then, since $\left(\theta_{3}+\theta_{2}\right)=\pi$, we can simplify (15) to

$$
Z_{04}+Z_{05} \cot \left(\theta_{5} / 2\right) \tan \theta_{4}=0
$$

Which is the second resonance condition given by (2) for the even-mode resonance of a typical stepped-impedance resonator [16].

\section{B. Odd -mode analysis}

In the odd mode, ports 2 and 3 are excited by signals of the same amplitude but of opposite phases. The introduction of equal and contrary voltages implies a diverging current that can be represented by Brazilian Microwave and Optoelectronics Society-SBMO received 23 June 2019; for review 27 June 2019 ; accepted 18 Nov 2019 
short circuits at the plane of symmetry which has the effect of introducing a virtual ground along the line of symmetry as shown in Fig. 7. In this analysis, we can choose $\theta_{2}=\theta_{3}=\pi / 2$ in order to have a symmetric design which will reduce the computational complexity. The calculation of the input impedance $Z_{\text {ino }}$ viewed from port 2 is simplified and corresponds to $Z_{\text {eq1 }}$ and $Z_{\text {eq2 }}$ connected in parallel.

From the transmission line theory, we can easily calculate $Z_{\text {eq } 1}$ as the input impedance of a shortcircuited line.

$$
Z_{e q 1}=j Z_{01} \tan \theta_{1}
$$

Referring to Fig. 7, the characteristic impedances $Z_{\text {ino1 }}$ and $Z_{\text {ino2 }}$ can be expressed as

$$
\begin{gathered}
Z_{\text {ino } 1}=j Z_{05} \tan \left(\theta_{5} / 2\right) \\
Z_{\text {ino } 2}=Z_{04} \frac{\left(Z_{\text {ino } 1}+j Z_{04} \tan \theta_{4}\right)}{\left(Z_{04}+j Z_{\text {ino } 1} \tan \theta_{4}\right)} \\
Z_{\text {ino } 2}=\frac{j Z_{04}\left(Z_{05} \tan \left(\theta_{5} / 2\right)+Z_{04} \tan \theta_{4}\right)}{\left(Z_{04}-Z_{05} \tan \left(\theta_{5} / 2\right) \tan \theta_{4}\right)} \\
Z_{\text {ino } 3}=\frac{Z_{03}{ }^{2}}{(R / 2)}=\frac{Z_{01}{ }^{2}}{(R / 2)}
\end{gathered}
$$

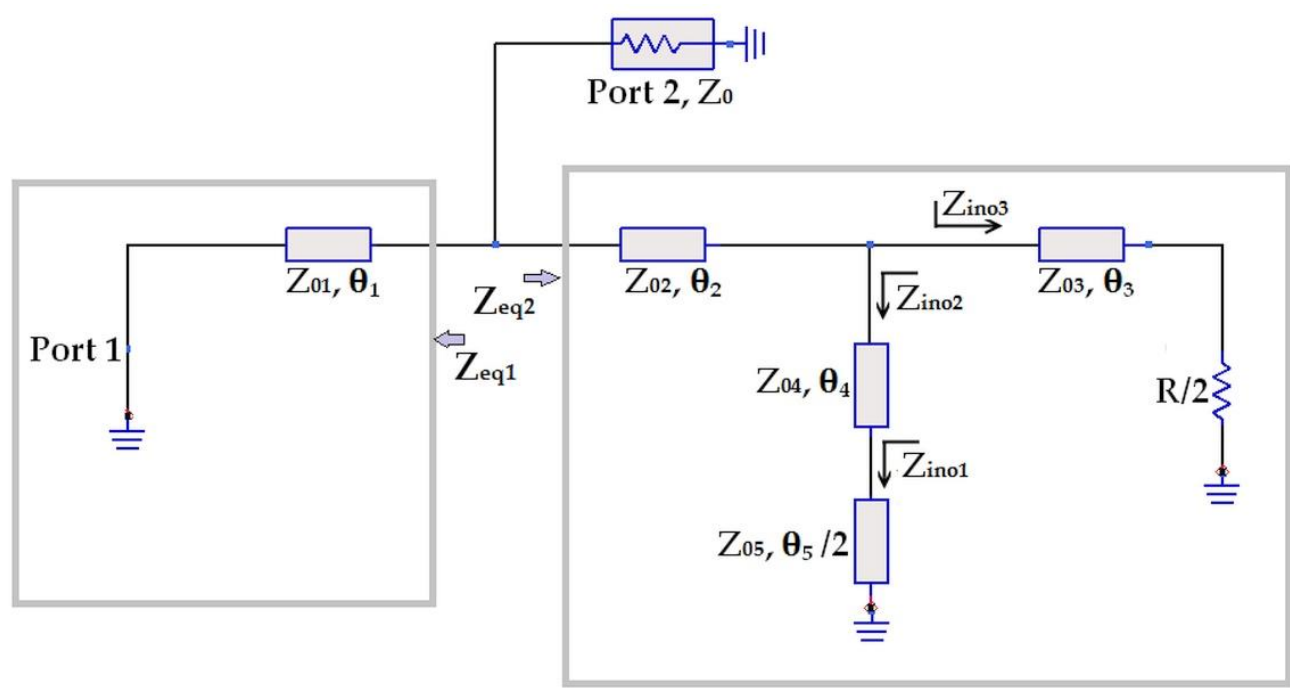

Fig. 7. Equivalent circuit of the proposed ring divider for even-mode.

The input impedance $Z_{\text {eq2 }}$ can be derived by using the quarter-wave transformer having characteristic impedance $\mathrm{Z}_{02}=\mathrm{Z}_{01}$, resulting in:

$$
\begin{gathered}
Z_{e q 2}=Z_{01}^{2}\left(Y_{\text {ino } 3}+Y_{\text {ino } 2}\right) \\
Z_{e q 2}=\frac{R}{2}-\frac{j Z_{01}^{2}\left(Z_{04}-Z_{05} \tan \left(\theta_{5} / 2\right) \tan \theta_{4}\right)}{Z_{04}\left(Z_{04} \tan \theta_{4}+Z_{05} \tan \left(\theta_{5} / 2\right)\right)}
\end{gathered}
$$

The input admittance $Y_{\text {ino }}$ seen from port 2 must be matched to $Y_{0}=1 / Z_{0}$. 


$$
\begin{gathered}
Y_{\text {ino }}=\frac{1}{Z_{e q 1}}+\frac{1}{Z_{e q 2}} \\
Y_{\text {ino }}=\frac{1}{j Z_{01} \tan \theta_{1}}+\frac{1}{\frac{R}{2}-\frac{j Z_{01}^{2}\left(Z_{04}-Z_{05} \tan \left(\theta_{5} / 2\right) \tan \theta_{4}\right)}{Z_{04}\left(Z_{04} \tan \theta_{4}+Z_{05} \tan \left(\theta_{5} / 2\right)\right)}=\frac{1}{Z_{0}}}
\end{gathered}
$$

Since $\theta_{1}=\pi / 2$ and $Z_{0}=R / 2$; then after simplification, the matching condition in equation (26) becomes:

$$
Z_{04}-Z_{05} \tan \left(\theta_{5} / 2\right) \tan \theta_{4}=0
$$

Which is the first resonance condition given by (1) for the odd-mode resonance of a typical steppedimpedance resonator. Since the two conditions for even and odd mode resonance are satisfied, this proves that the added element is an SIR.

The SIR is designed with fixed fundamental frequency, but different first spurious mode $\left(\mathrm{f}_{\mathrm{s} 1}\right)$ by choosing different combinations of $\mathrm{K}$ and $\alpha$ from Fig. 5 and thereby the dimensions of the SIR can be determined. The impedance ratio $\mathrm{K}$ and the stepped percentage $\alpha$ are then tuned using electromagnetic (EM) simulation in order to consider step discontinuities [16].

It has been demonstrated that by changing the width ratio $\mathrm{w}_{5} / \mathrm{w}_{4}$ of the low-impedance $\left(\mathrm{Z}_{05}\right)$ line to the high-impedance $\left(\mathrm{Z}_{04}\right)$ line, which result in the modification of $\mathrm{K}$, the position of the transmission zeros can be adjusted. Furthermore, it is evident that by increasing the width ratio $\mathrm{w}_{5} / \mathrm{w}_{4}$, resonance frequency is varied [17].

A parametric study is conducted to evaluate the effect of SIR's characteristic impedance ratio on the bandwidth of the proposed power divider. Fig. 8 shows the effect of variation of $\mathrm{K}$ on the transmission S-parameter magnitude of the proposed power divider.

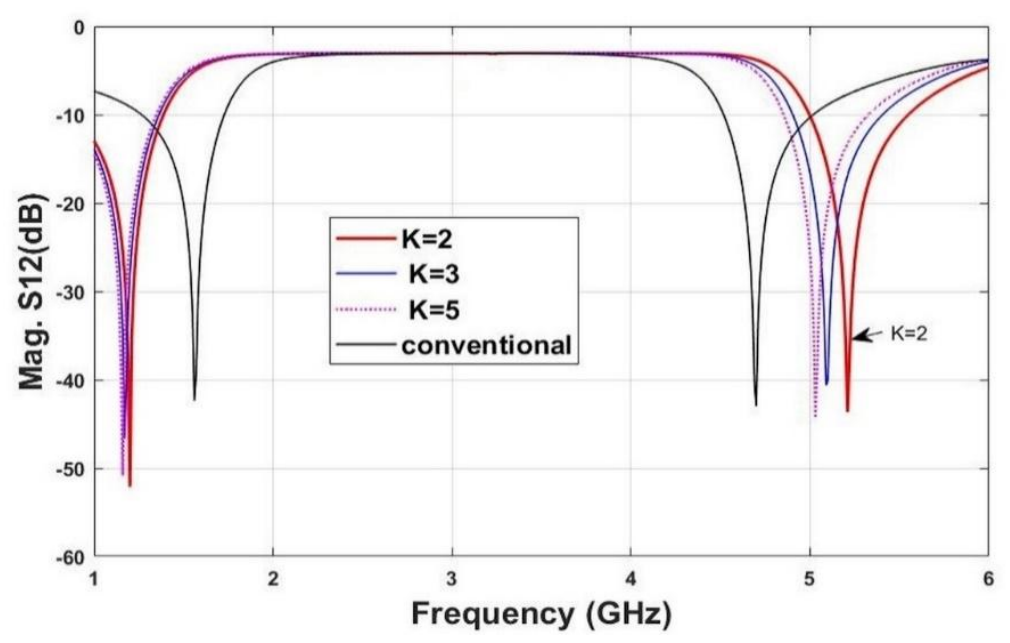

Fig. 8. Transmission S-parameter of proposed power divider with the variation of K.

It can be seen from Fig. 8, that when $\mathrm{K}$ increases, there is almost no effect on the lower transmission zero; however, the second transmission zero is shifted to a lower frequency. The 
simulation results are also compared to the case of the conventional ring power divider (without SIR). It is noticed that for $\mathrm{K}=2$, we get the wider bandwidth with two transmission zeros situated at 1.2 $\mathrm{GHz}$ and $5.21 \mathrm{GHz}$ with $\quad-52 \mathrm{~dB}$ and $-44 \mathrm{~dB}$ attenuation values respectively.

Following the above guidelines, the optimized parameters of $\mathrm{K}=2$ and $\alpha=0.95$ can be used in order to get a wide bandwidth from $1.7 \mathrm{GHz}$ to $4.6 \mathrm{GHz}$ of the proposed divider. As a result, the stepped impedance resonator can be designed to have the characteristic impedances $Z_{04}=40 \Omega$ and $Z_{05}=20 \Omega$, and the electrical lengths $\theta_{4}$ and $\theta_{5}$ are set to $84.5^{\circ}$ and $7.8^{\circ}$ respectively. This can be used in order to calculate the dimensions of the Stepped Impedance Resonator (SIR) cell. The microstrip widths and lengths corresponding to characteristic impedances $\mathrm{Z}_{0}, \mathrm{Z}_{01}, \mathrm{Z}_{04}$ and $\mathrm{Z}_{05}$ are shown in Table 1 . The radius of the ring is $15 \mathrm{~mm}$.

TABLE I. DIMENSIONS OF THE PROPOSED RING POWER DIVIDER

\begin{tabular}{|c|c|c|c|c|}
\hline Characteristic Impedance & $\mathrm{Z}_{0}$ & $\mathrm{Z}_{01}$ & $\mathrm{Z}_{04}$ & $\mathrm{Z}_{05}$ \\
\hline Width $(\mathrm{mm})$ & 0.76 & 0.44 & 0.89 & 2.2 \\
\hline Length $(\mathrm{mm})$ & 18.8 & 23 & 30 & 1.3 \\
\hline
\end{tabular}

Fig. 9 and Fig. 10 show the EM simulation results of the conventional and proposed ring power divider respectively obtained by using the Advanced Design System (ADS) software. As can be observed from these figures, the conventional ring structure [10] has a fractional bandwidth of $63 \%$ ranging from $2.14 \mathrm{GHz}$ to $4.11 \mathrm{GHz}\left(\mathrm{S}_{11}<-10 \mathrm{~dB}\right)$, while simulations of the proposed ring structure show that the fractional bandwidth has increased up to $90.2 \%$ producing therefore a remarkable bandwidth enhancement of $27.2 \%$ as mentioned in section 1. By comparing the results of the two ring power dividers, we can notice that the proposed ring power divider has an insertion loss which is between $-3.04 \mathrm{~dB}$ and $-3.58 \mathrm{~dB}$ over the whole band (1.74- $4.6 \mathrm{GHz}$ ) with quasi-null amplitude imbalance $\left(S_{21}=S_{31}\right)$ while the conventional power divider has an insertion loss between $-3.06 \mathrm{~dB}$ and $-3.61 \mathrm{~dB}$ over a narrower band (2.14- $4.11 \mathrm{GHz})$. In addition, it is obvious that the proposed divider has better return loss and better isolation between the output ports which are lower than $-10 \mathrm{~dB}$ in the whole band (1.74- $4.6 \mathrm{GHz}$ ), providing thus a significant wide bandwidth contribution compared to the previous reported ring divider which operates from $2.14 \mathrm{GHz}$ to $4.11 \mathrm{GHz}$ as can be clearly seen from Fig. 9 and Fig. 10.

Compared with other wideband power dividers present in the literature such as in [8]-[9], it is obvious that apart from its simpler structure, the proposed ring power divider has better performance over a wider operational bandwidth. It is to be noted that the power dividers developed in [8]-[9] have a fractional bandwidth of $68.6 \%$ and $62 \%$ respectively. 

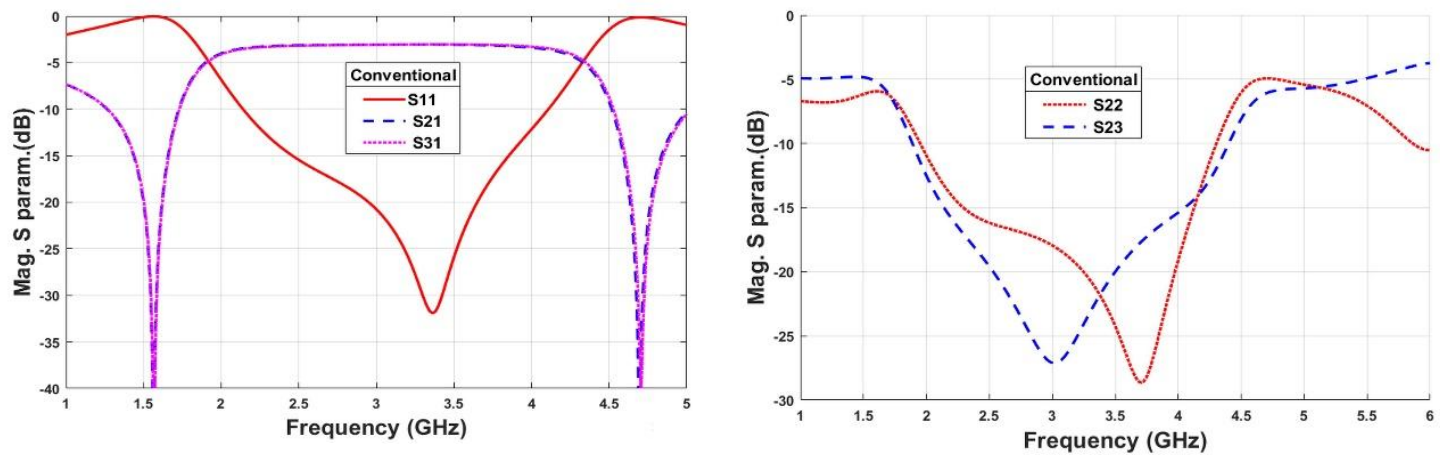

Fig. 9. Simulated S-parameters of the conventional power divider: (a) $S_{11}, S_{21} \& S_{31}$ and (b) $S_{22} \& S_{23}$.
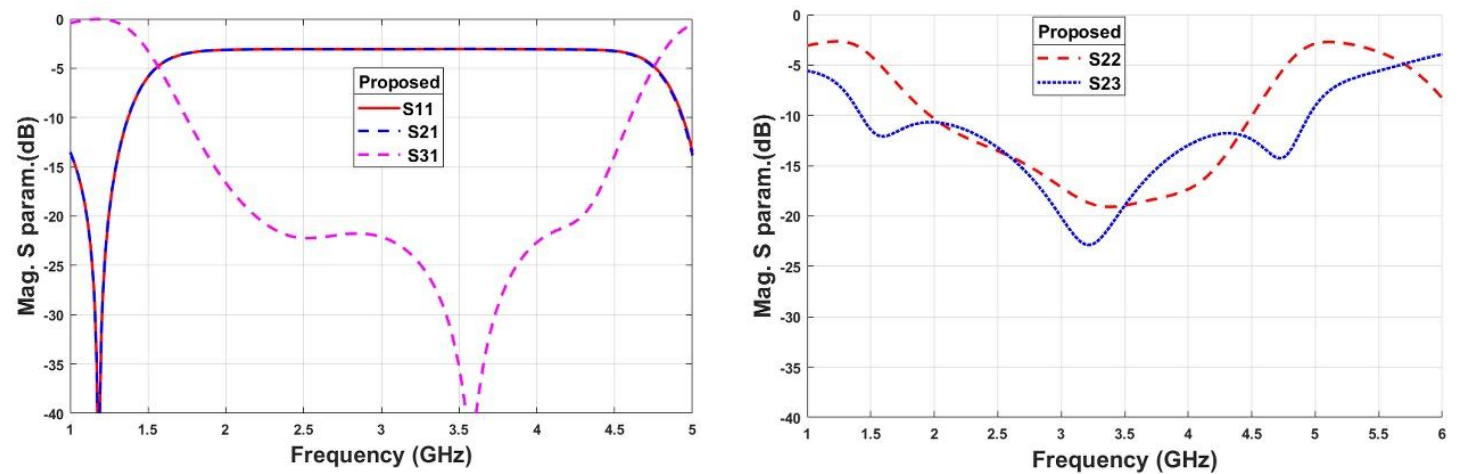

Fig. 10. Simulated S-parameters of the proposed power divider: (a) $S_{11}, S_{21} \& S_{31}$ and (b) $S_{22} \& S_{23}$.

\section{FABRICATION AND MEASUREMENT RESULTS}

For verification of the theoretical results, the proposed power divider has been fabricated on a Rogers RT/Duroid 6002 substrate whose dielectric constant is 2.93, loss tangent of 0.0013 and its thickness $0.254 \mathrm{~mm}$. It operates at the center frequency of $3.17 \mathrm{GHz}$ and has a large bandwidth (1.7$4.64 \mathrm{GHz})$ as expected. The fabricated ring power divider is shown in Fig. 11 and it occupies a small surface area of $5.1 \mathrm{~cm} \times 5.5 \mathrm{~cm}$.

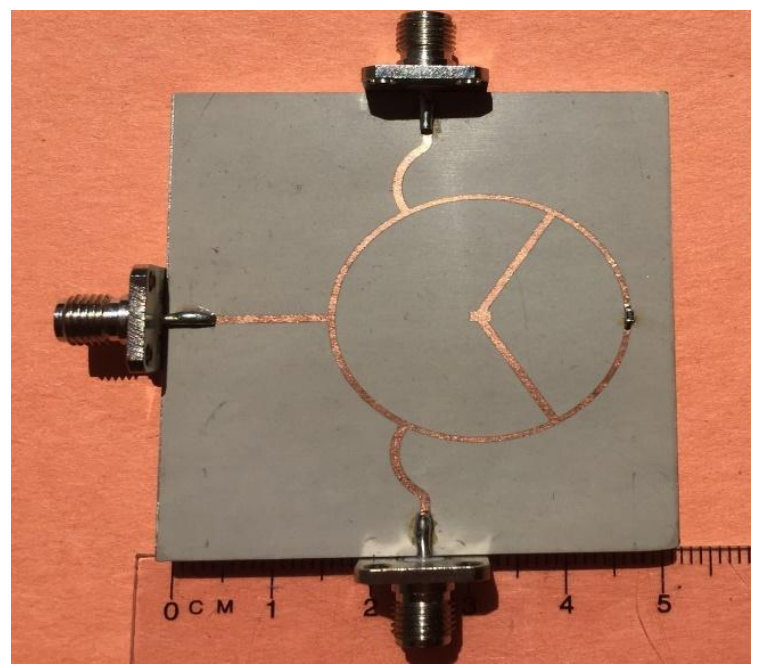

Fig. 11. Photograph of the fabricated ring power divider. 
The S-parameter measurements were accomplished using the Agilent E8362B vector network analyzer over the frequency range from 1 to $6 \mathrm{GHz}$. The EM simulations and measured S-parameter results of the proposed power divider are shown in Fig. 12 and Fig. 13. The measurement results show that the proposed power divider has an insertion loss of 0.74 ranging from $-3.36 \mathrm{~dB}$ to $-4.1 \mathrm{~dB}$ between $1.74 \mathrm{GHz}$ and $4.64 \mathrm{GHz}$. The measured return loss is less than $-10 \mathrm{~dB}$ from $1.6 \mathrm{GHz}$ to 4.71 GHz. The output return loss is lower than $-10 \mathrm{~dB}$ from $1.7 \mathrm{GHz}$ to $4.64 \mathrm{GHz}$. The isolation between the outputs is better than $-10 \mathrm{~dB}$ between $1.42 \mathrm{GHz}$ to $5.21 \mathrm{GHz}$. It can be concluded by comparing the measurement and EM simulation results that there is good agreement over the whole band of interest.

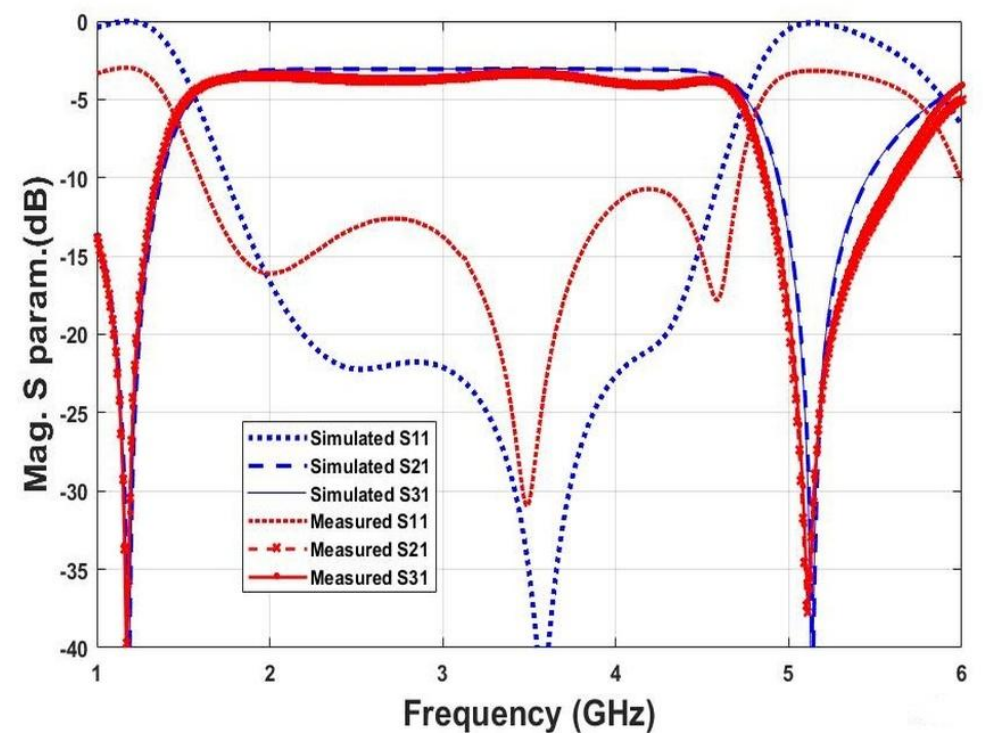

Fig. 12. Simulated and measured input return loss and insertion loss of the proposed divider.

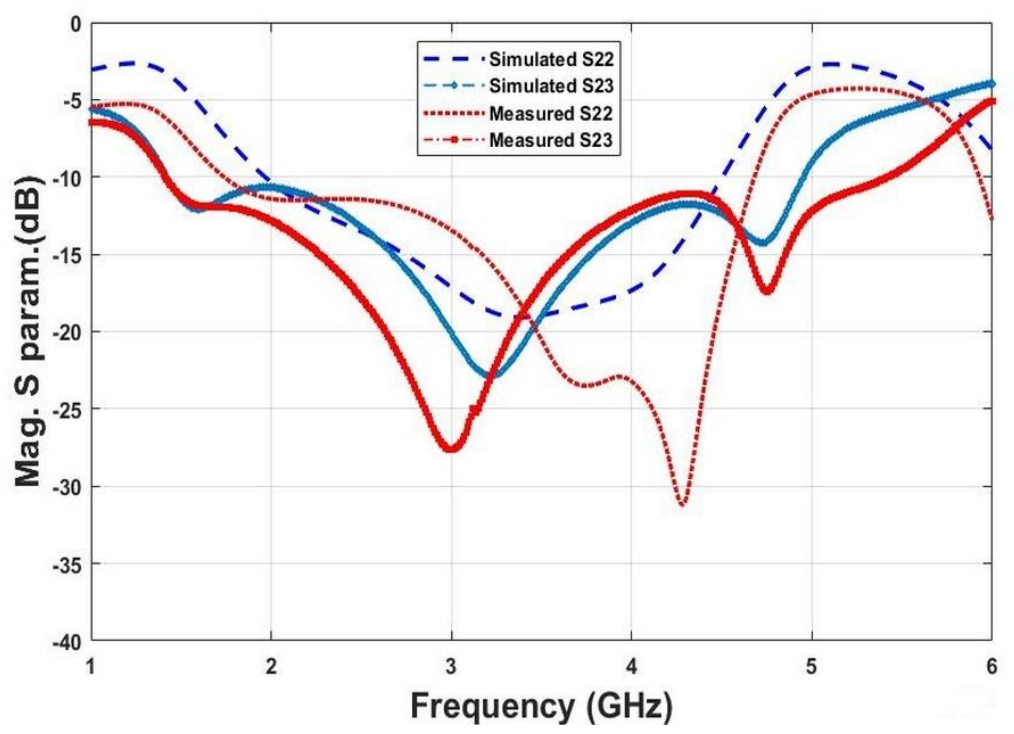

Fig. 13. Simulated and measured output return loss and isolation of the proposed divider.

The measured amplitude and phase imbalances between outputs of the proposed divider are shown in Fig. 14. These results show that the proposed power divider has a maximum amplitude imbalance Brazilian Microwave and Optoelectronics Society-SBMO received 23 June 2019; for review 27 June 2019; accepted 18 Nov 2019 Brazilian Society of Electromagnetism-SBMag 
of $0.21 \mathrm{~dB}$ in the whole band from $1.7 \mathrm{GHz}$ to $4.64 \mathrm{GHz}$ which implies that the power is divided evenly between the 2 outputs. In addition, the two outputs are in-phase in the whole band with a tolerance of less than 2.5 degrees over the whole bandwidth of interest which proves the good phase performance of the fabricated power divider.

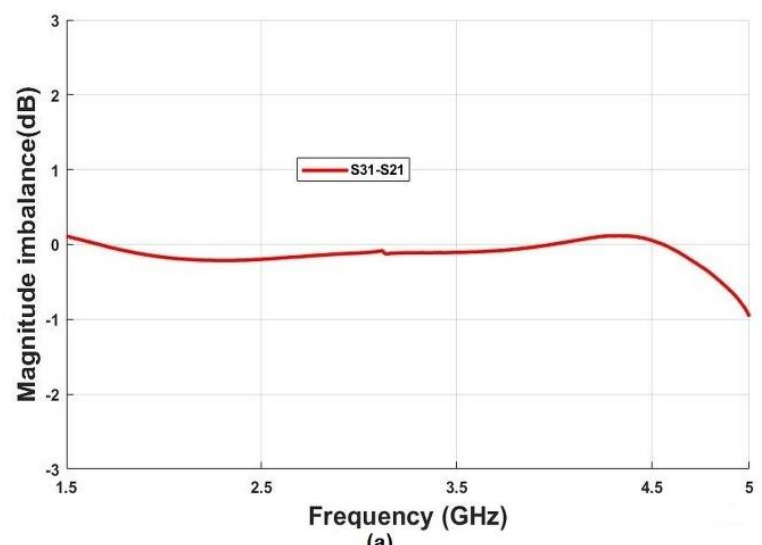

(a)

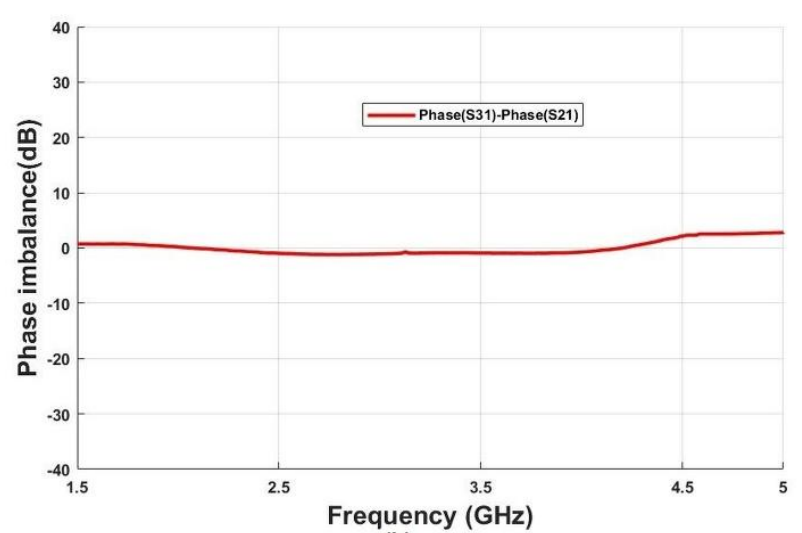

(b)

Fig. 14. Measured outputs imbalance of the proposed power divider: (a) Magnitude and (b) Phase.

\section{CONCLUSION}

In this work, we have designed a modified ring power divider to cover the frequency bandwidth of 1.7 GHz to $4.6 \mathrm{GHz}$ by adding an SIR to the old structure to improve its operational bandwidth. A detailed even-odd mode analysis of the proposed ring power divider has been performed. To validate the theoretical analysis and simulations, a prototype is fabricated on a single layer substrate. The experimental results show that the proposed circuit is broadband with a high factor amplitude balance over the considered bandwidth. The proposed ring power divider has the advantages of compact size and ease of fabrication which makes it very suitable for many wideband microwave applications.

\section{REFERENCES}

[1] E. Wilkinson, “An N-Way Hybrid Power Divider," IEEE Transactions on Microwave Theory and Techniques, vol. 8, no. 1, pp. 116-118, 1960.

[2] Y. Sun and A. Freundorfer, "Broadband folded Wilkinson power combiner/splitter," IEEE Microwave and Wireless Components Letters, vol. 14, no. 6, pp. 295-297, 2004.

[3] X.-P. Ou and Q.-X. Chu, "A modified two-section UWB Wilkinson power divider," 2008 International Conference on Microwave and Millimeter Wave Technology, 2008.

[4] B. Mishra, A. Rahman, S. Shaw, M. Mohd, S. Mondal, and P. P. Sarkar, "Design of an ultra-wideband Wilkinson power divider," 2014 First International Conference on Automation, Control, Energy and Systems (ACES), 2014.

[5] R. Pazoki, M. R. G. Fard, and H. G. Fard, "A Modification in the Single-Stage Wilkinson Power Divider to Obtain Wider Bandwidth," 2007 Asia-Pacific Microwave Conference, 2007.

[6] T.-J. Chang, T.-J. Huang, and H.-T. Hsu, "A new design of wilkinson power divider using radial stubs featuring size reduction and bandwidth enhancement with physical isolation," 2017 IEEE 18th Wireless and Microwave Technology Conference (WAMICON), 2017.

[7] M. Z. B. M. Nor, S. K. A. Rahim, M. I. B. Sabran, and M. S. B. A. Rani, "Wideband Planar Wilkinson Power Divider Using Double-Sided Parallel-Strip Line Technique,” Progress In Electromagnetics Research C, vol. 36, pp. 181-193, 2013.

[8] U. T. Ahmed and A. M. Abbosh, "Design of wideband single-layer in-phase power divider using microstrip to slotline coupled structure," Microwave and Optical Technology Letters, vol. 57, no. 4, pp. 789-791, 2015.

[9] S. S. Gao, S. Sun, and S. Xiao, "A Novel Wideband Bandpass Power Divider With Harmonic-Suppressed Ring Resonator," IEEE Microwave and Wireless Components Letters, vol. 23, no. 3, pp. 119-121, 2013.

[10] D. Hammou, E. Moldovan, and S. O. Tatu, "Novel MHMIC millimeter wave power divider/combiner," 2011 24th Canadian Conference on Electrical and Computer Engineering (CCECE), 2011.

Brazilian Microwave and Optoelectronics Society-SBMO received 23 June 2019; for review 27 June 2019; accepted 18 Nov 2019 
[11] D. Hammou, M. Nedil, and S. Tatu, "Design of improved ring Wilkinson power divider for millimetre wave applications," Electronics Letters, vol. 53, no. 8, pp. 542-544, 2017.

[12] A. Boutejdar, S. Elhani, S.D. Bennani, "Design of a novel slotted bandpass-bandstop filters using U-resonator and suspended multilayer-technique for L/X-band and Wlan/WiMax applications," International Conference on Electrical and Information Technologies (ICEIT), pp. 1-7, 2017.

[13] A. Boutejdar, M. Challal, S. Das, S. El Hani, "Design and Manufacturing of a Novel Compact 2.4 GHz LPF Using a DGS-DMS Combination and Quasi Octagonal Resonators for Radar and GPS Applications," Progress In Electromagnetics Research, vol. 90, pp. 15-28, 2019.

[14] A. Boutejdar, M. Challal, S. El Hani, "Design of a New Broad Stop Band (BSB) Lowpass Filter Using Compensated Capacitor and П-H-П DGS Resonator for Radar Applications," Progress In Electromagnetics Research, vol. 73, pp. 91$100,2018$.

[15] J.-T. Kuo and E. Shih, "Microstrip stepped impedance resonator bandpass filter with an extended optimal rejection bandwidth," IEEE Transactions on Microwave Theory and Techniques, vol. 51, no. 5, pp. 1554-1559, 2003.

[16] C.-Y. Hung, M.-H. Weng, Y.-K. Su, R.-Y. Yang, and H.-W. Wu, "Design of Compact and Sharp-Rejection UltraWideband Bandpass Filters Using Interdigital Stepped-Impedance Resonators," IEICE Transactions on Electronics, vol. E90-C, no. 8, pp. 1652-1654, 2007.

[17] L. Murmu, A. Bage, and S. Das, "Wideband bandpass filter using stepped impedance resonator with susceptibility test," Revue Roumaine des Sciences Techniques - Serie Électrotechnique et Énergétique, vol. 62, pp. 294-298, Oct. 2017.

[18] D. M. Pozar, Microwave engineering. New York: John Wiley \& Sons, 2011. 